\title{
Basal insulin reductions in anticipation of multiple exercise sessions in people with type 1 diabetes-a clinical perspective
}

\author{
Olivia McCarthy ${ }^{1,2}$, Steve C. Bain ${ }^{1}$, Rachel Deere ${ }^{1,2}$ \\ ${ }^{1}$ Diabetes Research Group Cymru, Medical School, Swansea University, Swansea, UK; ${ }^{2}$ Applied Sport, Technology, Exercise and Medicine Research \\ Centre (A-STEM), College of Engineering, Swansea University, Swansea, UK \\ Correspondence to: Olivia McCarthy. Applied Sport, Technology, Exercise and Medicine Research Centre (A-STEM), College of Engineering, Swansea \\ University, Bay campus, college of engineering, Fabian Way, Swansea SA1 8EN, UK. Email: 792328@swansea.ac.uk. \\ Provenance: This is an invited Editorial commissioned by the Section Editor Kaiping Zhang (AME College, AME Group, China). \\ Comment on: Moser O, Eckstein ML, Mueller A, et al. Reduction in insulin degludec dosing for multiple exercise sessions improves time spent in \\ euglycaemia in people with type 1 diabetes: A randomized crossover trial. Diabetes Obes Metab 2018. [Epub ahead of print].
}

Submitted Nov 23, 2018. Accepted for publication Nov 27, 2018.

doi: $10.21037 /$ atm.2018.11.63

View this article at: http://dx.doi.org/10.21037/atm.2018.11.63

\section{Normal physiology}

The islets of Langerhans are regions of the human pancreas that contain endocrine (hormone-producing) cells, constituting $1 \%$ to $2 \%$ of pancreas volume. There are four main types of endocrine cells within the islets of Langerhans: alpha $(\alpha)$ cells, beta $(\beta)$ cells, delta $(\delta)$ cells and to a lesser degree the pancreatic polypeptide (PP) cells. Of these populations, the $\beta$-cells account for between $65-80 \%$ of the pancreatic islets, and whilst predominately responsible for the secretion of the hormone insulin, they also co-secrete $\mathrm{C}$-peptide and amylin. Collectively, the cells in the islets of Langerhans are pivotal in glycaemic regulation and homeostasis.

The determinant of plasma glucose concentration is a function of the rate at which glucose enters the circulation (glucose appearance), balanced by the rate at which glucose is removed (glucose disappearance) (1). Circulating glucose originates from three sources; post-prandial intestinal absorption, glycogenolysis and gluconeogenesis. The attainment of glucose homeostasis requires a continuous interplay between several glucoregulatory hormones split via their ability to increase (glucagon, adrenaline, cortisol, growth hormone) or decrease (insulin) plasma glucose concentrations.

In regulatory metabolism, insulin is endogenously released in two phases. The first phase release occurs acutely and rapidly ( 10 minutes) in response to elevated circulating glucose levels. The second phase is independent of circulating glucose concentrations and occurs in a continuous, gradual release from the newly formed vesicles (2). Elevations in blood glucose increase the rate of insulin secretion from the $\beta$-cells. Once bound to its cell surface receptor, insulin facilitates the entry of extracellular glucose into the intracellular space, thereby lowering circulating levels of blood glucose. However, when glucose levels drop beyond those accepted within physiological norms, i.e., between 4-7 mmol/ $\mathrm{L}^{-1}$, insulin release slows or completely stops to avoid hypoglycaemia. A drop beyond this point $\left(\leq 3.6-3.9 \mathrm{mmol} / \mathrm{L}^{-1}\right)$ typically triggers the activation of several glucoregulatory hormones, i.e., glucagon and adrenaline which promote hepatic mediated glucose production via the process of glycogenolysis $(3,4)$.

\section{Type 1 diabetes (T1D)}

T1D is an autoimmune disease characterised by the progressive depletion and destruction of pancreatic $\beta$-cells, which results in hyperglycaemia and a propensity to develop diabetic ketoacidosis (DKA). However, whilst primarily associated with defective insulin secretory capacity, $\beta$-cell death also contributes to a progressive loss in $\alpha$-cell function (5). As a result, there is often a compromised functionality of glucagon, a peptide hormone which operates to raise glucose concentrations in response to low levels of circulating insulin. An impairment in these processes leads to an increased reliance on the 
third counter-regulatory mechanism, i.e., the release of adrenaline. As such attenuation or loss of this response can lead to hypoglycaemia unawareness, a condition which can have serious and sometimes fatal consequences (6).

\section{Exogenous insulin therapy}

Clinically, the inability of those with T1D to produce insulin necessitates a lifelong reliance on exogenous insulin therapy, typically administered by subcutaneous (SC) injection. Slight variations of the human insulin molecule are referred to insulin analogues. The term reflects the fact that they are not technically human insulin, rather they are analogues which retain the hormone's glucose management functionality.

When insulin is used in a clinical setting, a volume is injected into a site of SC tissue. The majority of existing insulin analogues are injected in hexameric form, however, hexamers are too large to readily pass through capillary fenestrae, and thus must first disassociate into either dimers or monomers within the injection depot before entering the circulation. In primary target tissues, the exogenously administered insulin molecules bind to and activate insulin receptors, thereby triggering cellular cascades that promote intracellular glucose uptake and utilisation.

The pharmacological production of insulin has progressed substantially since its discovery in 1921, with significant biochemical adjustments having been made to improve both pharmacokinetic $(\mathrm{PK})$ and pharmacodynamic profiles. The progression from purified animal to recombinant human insulin was made by Eli Lilly, who produced insulin Humulin in 1982. Since then, the variety of insulin analogues has expanded considerably, encompassing a range of products that differ in their duration of action, time of onset and peak effect. The type of insulin analogue largely reflects its absorption rate, thus insulins are classified in relation to their duration of action, i.e., ultra-fast acting, fast-acting, short-acting, intermediateacting, long-acting, ultra-long acting, and combination products (Table 1).

\section{Developments in basal insulin analogues}

In the case of basal insulin development, the challenge has been to retard absorption to a greater extent than occurs with human insulin-ideally to produce a constant, peakless kinetic profile that more mimics normal basal insulin secretion (16).
Unlike endogenous insulin production (which is secreted in a glucose-dependent manner), the dose of exogenous insulin must be manually titrated to maintain euglycemia (17). As such, correctly identifying a dosing strategy across a 24-hour time span is an important feature for minimising within-patient variability (17). However, basal analogues have presented a number of flaws which compromise the attainment of this goal. Produced back in the 1950's, the original human insulin-based products, such as NPH insulin, had suboptimal PK profiles, characterised by inappropriate peaks of action coupled with high variability from injection to injection. Combined, these attributes not only accentuated the risk of hypoglycaemia but also necessitated the need for twice daily dosing.

In the early 2000's, insulins detemir (IDet) $\left(\right.$ Levemir $^{\mathrm{TM}}$ ) and glargine (Lantus ${ }^{\mathrm{TM}}$ ) were created in an attempt to overcome some of the $\mathrm{PK}$ issues of their preceding analogues.

However, in comparison to regular human insulin, neither provide an ideal absorption profile that closely mimics normal physiology (16). Thus, the development of ultra-long acting insulins that achieves steady state (SS) PK with a low peak: trough ratio should be of clinical and patient advantage.

\section{Insulin Glargine (IGlar) 300 units/mL}

IGlar substitutes the amino group $21^{\mathrm{A}}$-Asn for Gly thereby forming its unique chemical structure $\left(21^{\mathrm{A}}\right.$-Gly$31^{\mathrm{B}}$-Arg-32 $2^{\mathrm{B}}$-Arg-human insulin) (15). Following SC injection, IGlar precipitates at physiological $\mathrm{pH}$ to form a depot from which IGlar is slowly released (18). In 2015, a more concentrated form of IGlar [IGlar 300 units/mL (IGlar U300)] was released. IGlar U300 has a reduced redissolution rate, longer duration of action and lower fluctuation rate following SC injection compared to glargine 100 units/mL (U100) (19). However, whilst its PK and pharmacodynamic profile is improved, ultrastructural three-dimensional research has observed the formation of larger microprecipitates in the tissues following IGlar U300 injection. Physiologically, this may cause a disproportionate release and delayed absorption rate of insulin into the blood stream (20).

\section{Insulin degludec (IDeg)}

Recently, the development of IDeg; a new ultra-long acting insulin analogue with a duration of action that comfortably 
Table 1 Synopsis of currently available insulin analogues for individuals with type 1 diabetes

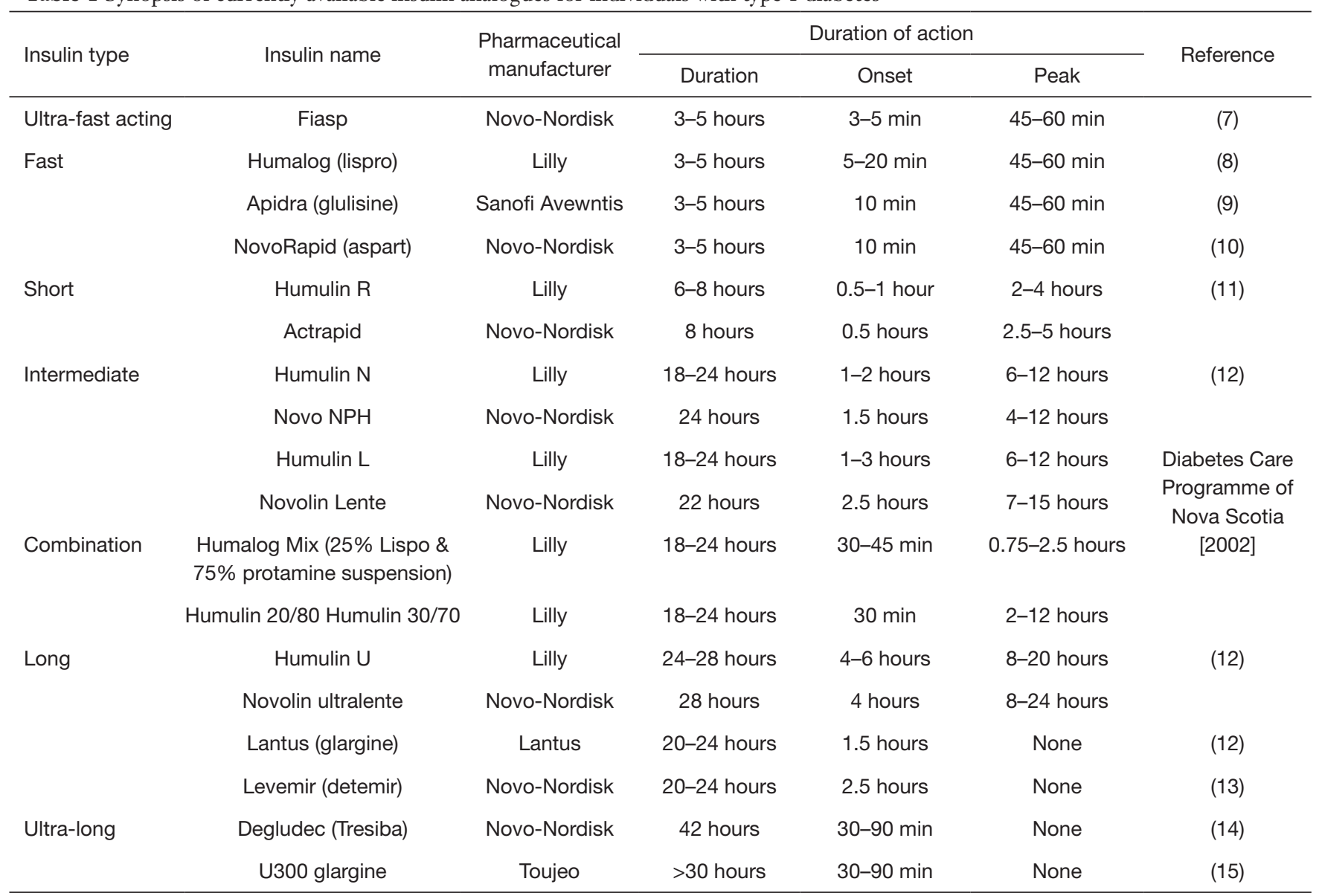

exceeds 24hours, has been achieved (17). The name "De-gluc-dec" originates from its unique structural profile which acylates DesB30 at the $\varepsilon$-amino group of LysB29 with a glutamic acid ligand at position 29. The opposing end of this glutamic acid 'spacer' is then attached to a hexadecandioyl 16 fatty diacid side chain, completing the biochemical structure of IDeg.

\section{Pharmacological profile}

The impressive pharmacological profile of IDeg derives from its specifically engineered molecular structure which encourages multi-hexamer formation via a prolonged depot release (17). To date, IDeg is the only insulin analogue to self-associate into multi-hexamers upon SC injection. In the pharmaceutical formulation, i.e., in the presence of phenyl and zinc, the IDeg hexamers adopt a formation from which only one hexameric end can interact with the side chain of another (17). After injection, the phenol is lost and the dihexamer poles open at both ends thereby enabling the formation of long soluble multi-hexamer chains (21). The loss of zinc allows the monomers to be slowly released which adeptly creates a soluble depot from which IDeg is gradually absorbed into the circulation on a continuous basis.

\section{Pharmacokinetic profile}

The ultra-long profile of IDeg brings with it several clinically relevant glycaemic outcomes. Firstly, research has shown that SS concentrations of IDeg are reached within 3 days of once-daily dosing with no risk of insulin 'stacking' (22). Moreover, concentration-time curves and glucose infusion rate (GIR) profiles obtained during a one-dosing interval at SS conditions have highlighted the consistent distribution of IDeg formulations over a typical $24 \mathrm{~h}$ treatment interval (23).

The flat shape of the pharmacodynamic profile of IDeg is supported by parameters such as distribution of the glucose lowering effect and relative fluctuation (17). Importantly, 
these characteristics support a glucose lowering effect across a range of clinically relevant dose levels $(0.4,0.6$ or $0.8 \mathrm{U} / \mathrm{kg}$ ) (24). Moreover, recent comparative research investigating glycaemic outcomes associated with IDeg versus IGlar U300, found IDeg produced a lower day-today and within-day variability than that of IGlar U300 in patients with T1D (20). Finally, the peak-less, extended and highly predictable glucose lowering profile of IDeg allows for variation in the once-daily-dosing strategy of up to 8 hours $(25,26)$ which has important social and practical patient appeals (27).

\section{Clinical questions and concerns}

The new generation of ultra-long basal insulins provide improved therapeutic care for patients with T1D. However, although progressive, the introduction of any new product demands careful consideration of important clinical outcomes. Similar to that of its preceding analogues, the efficacy of IDeg in minimising the occurrence and magnitude of hypoglycaemic requires thorough investigation. Unsurprisingly, both iatrogenic and exercise induced hypoglycaemia represent major concerns in this patient population. Thus, considering the heightened risk of developing hypoglycaemia both during and as long as 24 hours after exercise participation $(28,29)$, insulin management must be carefully considered.

During exercise, the increased skeletal muscle contraction rates, heightened insulin sensitivity and inability to regulate circulating insulin concentration result in an augmented skeletal muscle uptake but decreased appearance of circulating blood glucose. These physiological responses, coupled with the more rapid appearance of exogenous insulin due to elevations in both thermo-and-hemodynamics as well as an inability to effectively counteract the dropin glucose concentrations, exacerbate the likelihood of hypoglycaemia in individuals with T1D.

Therefore, although the importance of regular physical exercise in glucose management for individuals with T1D is magnified by its acknowledgement as a key adjunct to insulin therapy by several governmental, charitable and healthcare organisations-exercise participation rates remain low, with fears around loss of glycaemic control during and after exercise reported as one of the major barriers to regular engagement (30).

These fears are perhaps exacerbated by uncertainty in how to appropriately adjust exogenous insulins around exercise to best preserve blood glucose concentrations.
Whilst research has highlighted improved post exercise glycaemia when bolus insulin is reduced in the pre-andpost-exercise period (31), adjustments have mostly been done against a background of the basal insulins IGlar, NPH or IDet (32-39) with less work investigating IDeg.

For the majority of patients, basal insulins are not normally adjusted in anticipation of exercise. However, complete suspension of basal insulin administration in pump users during exercise has been found to decrease exercise-induced hypoglycaemia by $\sim 30 \%$ in children and adolescents (40). Although an effective strategy for pump users, complete basal omission in those using multiple daily injections (MDIs) is difficult and potentially dangerous. Encouragingly, recent work reported complete protection from exercise-induced and nocturnal episodes of hypoglycaemia when patients on MDI reduced basal insulin IGlar and IDet by $20 \%$ the morning of an anticipated exercise session in combination with a $75 \%$ reduction in rapid-acting insulin (100\% dose $=9,80 \%$ dose $=0)(41)$.

Whilst still in its infancy, research has started to explore glycaemic outcomes associated with exercise and/or bolus insulin manipulations on a background of IDeg in patients with T1D. Initial work by Moser and colleagues reported no hypoglycaemic episodes both during and after high intensity interval-and-continuous-exercise when multiple adjustments in bolus insulin were made on a background of stable IDeg dosing (42). More recently, comparative work has highlighted the low and similar risk of exercise-induced hypoglycaemia on a background of IDeg and IGlar (43). However, whilst IDeg has been used as the basal insulin, these studies have only involved acute manipulations to preexercise bolus insulin with no basal adjustments being made. Addressing this gap, Moser et al. was the first to experiment with IDeg adjustments around exercise in individuals with T1D (44).

\section{Study description}

Nine T1D individuals on a background of IDeg were recruited into the study. The authors set out to assess the effects of a reduction in IDeg around multiple aerobic exercise sessions at a moderate intensity $\left(63 \% \pm 7 \% \mathrm{VO}_{2 \max }\right)$. Basal insulin dose reductions were introduced 3 days before the first exercise session, with 5 consecutive days of exercise being performed under either a $100 \%$ or $75 \%$ individualised IDeg dose. Trials were separated by a 4 -week wash out period and acutely standardised with participants consuming a comparable $\sim 63 \mathrm{~g} \mathrm{CHO}$ at least two hours before the 
start of exercise combined with an individualised bolus insulin dose. This minimised the possible contributions of opposing basal and acute bolus insulin manipulations on exercise performance and glycaemia.

The two main variables assessed were (I) time spent in specific glycaemic ranges and (II) number of hypoglycaemic events over the five days of consecutive exercise. The study concluded that a $25 \%$ reduction of basal IDeg around exercise has the potential to increase time spent in euglycaemia, though had no influence on time spent in hyper- or hypoglycaemia or the number of hypoglycaemic episodes.

\section{Critique}

This study provided an excellent insight into acute glycaemic outcomes associated with IDeg manipulations around exercise. However, subject numbers were small and included a relatively homogenous group with good glycaemic control. This not only makes wider generalisation difficult, but also overlooks important diabetes related variables. Moreover, the dose reduction was made three days prior to the initial exercise session. Outside of elite or specifically structure sports programming, this degree of forewarning is largely unrealistic for the majority of recreationally active individuals. Thus, given the ultralong duration of IDeg, questions remain as to whether a reduction in the basal insulin dose on the day of, or evening before the anticipated exercise provides protection against the risk of hypoglycaemia.

Finally, although overall time spent in glycaemic ranges provides valuable metrics, a further breakdown focusing on specific time frames, i.e., acutely post exercise and nocturnal periods, would be of much clinical and practical relevance.

\section{Further research going forward}

With new insulin analogues that differ in their PK and pharmacodynamic profile being made available, exploring the interaction of modern insulin analogues and exercise is crucial in our understanding of how we can encourage patients with T1D to maintain a physically active lifestyle. The results of this study have set a premise for future research which should continue to investigate the effect of IDeg manipulations around exercise, carbohydrate feeding and rapid acting insulin adjustments. From a clinical perspective the inclusion of patients with varying degrees of glycaemic control and physical fitness would provide invaluable information that assists the development of exercise guidelines.

\section{Acknowledgements}

None.

\section{Footnote}

Conflicts of Interest: The authors have no conflicts of interest to declare.

\section{References}

1. Aronoff SL, Berkowitz K, Shreiner B, et al. Glucose Metabolism and Regulation: Beyond Insulin and Glucagon. Diabetes Spectrum 2004;17:183-90.

2. Schuit F, Moens K, Heimberg H, et al. Cellular Origin of Hexokinase in Pancreatic Islets. J Biol Chem 1999;274:32803-9.

3. Schwartz NS, Clutter WE, Shah SD, et al. Glycemic thresholds for activation of glucose counterregulatory systems are higher than the threshold for symptoms. J Clin Invest 1987;79:777-81.

4. American Diabetes Association. Defining and reporting hypoglycemia in diabetes: a report from the American Diabetes Association Workgroup on Hypoglycemia. Diabetes Care 2005;28:1245-9.

5. Banarer S, McGregor VP, Cryer PE. Intraislet Hyperinsulinemia Prevents the Glucagon Response to Hypoglycemia Despite an Intact Autonomic Response. Diabetes 2002;51:958-65.

6. Verberne AJ, Korim WS, Sabetghadam A, et al. Adrenaline: insights into its metabolic roles in hypoglycaemia and diabetes. Br J Pharmacol 2016;173:1425-37.

7. Hövelmann U, Heise T, Nosek L, et al. Pharmacokinetic Properties of Fast-Acting Insulin Aspart Administered in Different Subcutaneous Injection Regions. Clin Drug Investig 2017;37:503-9.

8. Howey DC, Bowsher RR, Brunelle RL, et al. [Lys(B28), Pro(B29)]-human insulin. A rapidly absorbed analogue of human insulin. Diabetes 1994;43:396-402.

9. Danne T, Becker RH, Heise T, et al. Pharmacokinetics, prandial glucose control, and safety of insulin glulisine in children and adolescents with type 1 diabetes. Diabetes Care 2005;28:2100-5.

10. Plank J, Wutte A, Brunner G, et al. A direct comparison of insulin aspart and insulin lispro in patients with type 1 
diabetes. Diabetes Care 2002;25:2053-7.

11. Woodworth JR, Howey DC, Bowsher RR. Establishment of time-action profiles for regular and NPH insulin using pharmacodynamic modeling. Diabetes Care 1994;17:64-9.

12. Lepore M, Pampanelli S, Fanelli C, et al. Pharmacokinetics and pharmacodynamics of subcutaneous injection of longacting human insulin analog glargine, NPH insulin, and ultralente human insulin and continuous subcutaneous infusion of insulin lispro. Diabetes 2000;49:2142-8.

13. Porcellati F, Rossetti P, Busciantella NR, et al. Comparison of Pharmacokinetics and Dynamics of the Long-Acting Insulin Analogs Glargine and Detemir at Steady State in Type 1 Diabetes: A double-blind, randomized, crossover study. Diabetes Care 2007;30:2447-52.

14. Nasrallah SN, Reynolds LR. Insulin Degludec, The New Generation Basal Insulin or Just another Basal Insulin? Clin Med Insights Endocrinol Diabetes 2012;5:31-7.

15. Becker RH, Dahmen R, Bergmann K, et al. New insulin glargine 300 Units $\cdot \mathrm{mL}-1$ provides a more even activity profile and prolonged glycemic control at steady state compared with insulin glargine 100 Units $\cdot \mathrm{mL}-1$. Diabetes Care 2015;38:637-43.

16. Goldman J, Kapitza C, Pettus J, et al. Understanding how pharmacokinetic and pharmacodynamic differences of basal analog insulins influence clinical practice. Curr Med Res Opin 2017;33:1821-31.

17. Haahr H, Heise T. A Review of the Pharmacological Properties of Insulin Degludec and Their Clinical Relevance. Clin Pharmacokinet 2014;53:787-800.

18. Kuerzel GU, Shukla U, Scholtz HE, et al. Biotransformation of insulin glargine after subcutaneous injection in healthy subjects. Curr Med Res Opin 2003;19:34-40.

19. Lamos EM, Younk LM, Davis SN. Concentrated insulins: the new basal insulins. Ther Clin Risk Manag 2016;12:389-400

20. Heise T, Nørskov M, Nosek L, et al. Insulin degludec: Lower day-to-day and within-day variability in pharmacodynamic response compared with insulin glargine $300 \mathrm{U} / \mathrm{mL}$ in type 1 diabetes. Diabetes Obes Metab 2017;19:1032-9.

21. Jonassen I, Havelund S, Hoeg-Jensen T, et al. Design of the Novel Protraction Mechanism of Insulin Degludec, an Ultra-long-Acting Basal Insulin. Pharm Res 2012;29:2104-14.

22. Heise T, Meneghini L. Insulin Stacking Versus Therapeutic Accumulation: Understanding the Differences. Endocr Pract 2014;20:75-83.
23. Korsatko S, Deller S, Mader JK, et al. Ultra-Long Pharmacokinetic Properties of Insulin Degludec are Comparable in Elderly Subjects and Younger Adults with Type 1 Diabetes Mellitus. Drugs Aging 2014;31:47-53.

24. Heise T, Hermanski L, Nosek L, et al. Insulin degludec: four times lower pharmacodynamic variability than insulin glargine under steady-state conditions in type 1 diabetes. Diabetes Obes Metab 2012;14:859-64.

25. Mathieu C, Hollander P, Miranda-Palma B, et al. Efficacy and Safety of Insulin Degludec in a Flexible Dosing Regimen vs Insulin Glargine in Patients With Type 1 Diabetes (BEGIN: Flex T1): A 26-Week Randomized, Treat-to-Target Trial With a 26-Week Extension. J Clin Endocrinol Metab 2013;98:1154-62.

26. Meneghini L, Atkin SL, Gough SC, et al. The Efficacy and Safety of Insulin Degludec Given in Variable OnceDaily Dosing Intervals Compared With Insulin Glargine and Insulin Degludec Dosed at the Same Time Daily: A 26-week, randomized, open-label, parallel-group, treatto-target trial in individuals with type 2 diabetes. Diabetes Care 2013;36:858-64.

27. Josse RG, Woo V. Flexibly timed once-daily dosing with degludec: a new ultra-long-acting basal insulin. Diabetes Obes Metab 2013;15:1077-84.

28. Steppel JH, Horton ES. Exercise in the management of type 1 diabetes mellitus. Rev Endocr Metab Disord 2003;4:355-60.

29. Tsalikian E, Mauras N, Beck RW, et al. Impact of Exercise on Overnight Glycemic Control in Children with Type 1 Diabetes Mellitus. J Pediatr 2005;147:528-34.

30. Brazeau AS, Rabasa-Lhoret R, Strychar I, et al. Barriers to physical activity among patients with type 1 diabetes. Diabetes Care 2008;31:2108-9.

31. Campbell MD, Walker M, Trenell MI, et al. Large pre- and postexercise rapid-acting insulin reductions preserve glycemia and prevent early- but not late-onset hypoglycemia in patients with type 1 diabetes. Diabetes Care 2013;36:2217-24.

32. Campaigne BN, Wallberg-Henriksson H, Gunnarsson R. Glucose and insulin responses in relation to insulin dose and caloric intake $12 \mathrm{~h}$ after acute physical exercise in men with IDDM. Diabetes Care 1987;10:716-21.

33. De Feo P, Di Loreto C, Ranchelli A, et al. Exercise and diabetes. Acta Biomed 2006;77 Suppl 1:14-7.

34. Nagi D. editor. Exercise and Sport in Diabetes 2nd Edition. Chichester, UK: John Wiley \& Sons, Ltd., 2005.

35. Mauvais-Jarvis F, Sobngwi E, Porcher R, et al. Glucose response to intense aerobic exercise in type 1 diabetes: 
maintenance of near euglycemia despite a drastic decrease in insulin dose. Diabetes Care 2003;26:1316-7.

36. Rabasa-Lhoret R, Bourque J, Ducros F, et al. Guidelines for premeal insulin dose reduction for postprandial exercise of different intensities and durations in type 1 diabetic subjects treated intensively with a basal-bolus insulin regimen (ultralente-lispro). Diabetes Care 2001;24:625-30.

37. West DJ, Morton RD, Bain SC, et al. Blood glucose responses to reductions in pre-exercise rapid-acting insulin for $24 \mathrm{~h}$ after running in individuals with type 1 diabetes. J Sports Sci 2010;28:781-8.

38. West DJ, Stephens JW, Bain SC, et al. A combined insulin reduction and carbohydrate feeding strategy $30 \mathrm{~min}$ before running best preserves blood glucose concentration after exercise through improved fuel oxidation in type 1 diabetes mellitus. J Sports Sci 2011;29:279-89.

39. Campbell MD, Walker M, Trenell MI, et al. Metabolic Implications when Employing Heavy Pre- and PostExercise Rapid-Acting Insulin Reductions to Prevent Hypoglycaemia in Type 1 Diabetes Patients: A Randomised Clinical Trial. PLoS One 2014;9:e97143.

40. Tsalikian E, Kollman C, Tamborlane WB, et al. Prevention of hypoglycemia during exercise in children with type

Cite this article as: McCarthy O, Bain SC, Deere R. Basal insulin reductions in anticipation of multiple exercise sessions in people with type 1 diabetes-a clinical perspective. Ann Transl Med 2018;6(Suppl 2):S111. doi: 10.21037/atm.2018.11.63
1 diabetes by suspending basal insulin. Diabetes Care 2006;29:2200-4.

41. Campbell MD, Walker M, Bracken RM, et al. Insulin therapy and dietary adjustments to normalize glycemia and prevent nocturnal hypoglycemia after evening exercise in type 1 diabetes: a randomized controlled trial. BMJ Open Diabetes Res Care 2015;3:e000085.

42. Moser O, Tschakert G, Mueller A, et al. Effects of HighIntensity Interval Exercise versus Moderate Continuous Exercise on Glucose Homeostasis and Hormone Response in Patients with Type 1 Diabetes Mellitus Using Novel Ultra-Long-Acting Insulin. PLoS One 2015;10:e0136489.

43. Heise T, Bain SC, Bracken RM, et al. Similar risk of exercise-related hypoglycaemia for insulin degludec to that for insulin glargine in patients with type 1 diabetes: a randomized cross-over trial. Diabetes Obes Metab 2016;18:196-9.

44. Moser O, Eckstein ML, Mueller A, et al. Reduction in insulin degludec dosing for multiple exercise sessions improves time spent in euglycaemia in people with type 1 diabetes: a randomised cross-over trial. Diabetes Obes Metab 2018. [Epub ahead of print]. 